\title{
Pediatric Patients Demonstrate Progressive T1-Weighted Hyperintensity in the Dentate Nucleus following Multiple Doses of Gadolinium-Based Contrast Agent
}

(DD.R. Roberts, (D)A.R. Chatterjee, (DM. Yazdani, DB. Marebwa, (D). Brown, (DH. Collins, (D) Bolles, (D).M. Jenrette, (D)P.J. Nietert, and (D)X. Zhu

\begin{abstract}
BACKGROUND AND PURPOSE: While there have been recent reports of brain retention of gadolinium following gadolinium-based contrast agent administration in adults, a retrospective series of pediatric patients has not previously been reported, to our knowledge. We investigated the relationship between the number of prior gadolinium-based contrast agent doses and increasing $\mathrm{Tl}$ signal in the dentate nucleus on unenhanced T1-weighted MR imaging. We hypothesized that despite differences in pediatric physiology and the smaller gadolinium-based contrast agent doses that pediatric patients are typically administered based on weighted-adjusted dosing, the pediatric brain would also demonstrate dose-dependent increasing $\mathrm{Tl}$ signal in the dentate nucleus.
\end{abstract}

MATERIALS AND METHODS: We included children with multiple gadolinium-based contrast agent administrations at our institution. A blinded reader placed ROIs within the dentate nucleus and adjacent cerebellar white matter. To eliminate reader bias, we also performed automated ROI delineation of the dentate nucleus, cerebellar white matter, and pons. Dentate-to-cerebellar white matter and dentate-to pons ratios were compared with the number of gadolinium-based contrast agent administrations.

RESULTS: During 20 years at our institution, 280 patients received at least 5 gadolinium-based contrast agent doses, with 1 patient receiving 38 doses. Sixteen patients met the inclusion/exclusion criteria for ROI analysis. Blinded reader dentate-to-cerebellar white matter ratios were significantly associated with gadolinium-based contrast agent doses $\left(r_{\mathrm{s}}=0.77, P=.001\right)$. The dentate-to-pons ratio and dentate-to-cerebellar white matter ratios based on automated ROI placement were also significantly correlated with gadolinium-based contrast agent doses ( $t=4.98, P<.0001$ and $t=2.73, P<.02$, respectively).

CONCLUSIONS: In pediatric patients, the number of prior gadolinium-based contrast agent doses is significantly correlated with progressive T1-weighted dentate hyperintensity. Definitive confirmation of gadolinium deposition requires tissue analysis. Any potential clinical sequelae of gadolinium retention in the developing brain are unknown. Given this uncertainty, we suggest taking a cautious stance, including the use, in pediatric patients, of higher stability, macrocyclic agents, which in both human and animal studies have been shown to be associated with lower levels of gadolinium deposition, and detailed documentation of dosing. Most important, a patient should not be deprived of a well-indicated contrasted MR examination.

ABBREVIATIONS: DN/C = dentate-to-cerebellar white matter; DN/P = dentate-to-pons; GBCA = gadolinium-based contrast agent; MNI = Montreal Neurological Institute; SUIT = Spatially Unbiased Atlas Template of the Cerebellum and Brain Stem

G adolinium-based contrast agents (GBCAs) have been used extensively in the pediatric population for the evaluation of various central nervous system and non-CNS pathologies. The American College of Radiology Appropriateness Criteria, a set of evidence-based guidelines developed by the American College of

Received May 2, 2016; accepted after revision June 13

From the Departments of Radiology and Radiological Sciences (D.R.R., A.R.C., M.Y. B.M., T.B., H.C., G.B.), Neurosciences and Neuroscience Research (D.R.R.), Radiation Oncology (J.M.J.), Biostatistics (P.J.N.), and Public Health Sciences (P.J.N.), Medical University of South Carolina, Charleston, South Carolina; and Department of Psychology (X.Z.), Normal College, Shihezi University, Xinjiang, China.

This work was funded, in part, by the National Institutes of Health, National Center for Advancing Translational Science (grant No. ULITR001450).
Radiology to assist physician decision-making, considers postcontrast MR imaging appropriate in several pediatric clinical scenarios. ${ }^{1}$ The contrast agents available for use in children in the United States have FDA approval only for CNS indications, and several agents are not approved for pediatric use. At present only 1 GBCA (gadoteridol) is approved for children younger than 2

Paper previously presented at: Annual Meeting of the American Society of Neuroradiology and the Foundation of the ASNR Symposium, May 23-26, 2016; Washington, DC. Please address correspondence to Donna R. Roberts, MD, Department of Radiology and Radiological Sciences, 96 Jonathan Lucas St, MSC 323, Medical University of South Carolina, Charleston, SC 29425-3230; e-mail: robertdr@musc.edu

- Indicates open access to non-subscribers at www.ajnr.org

http://dx.doi.org/10.3174/ajnr.A4891 
years of age. Therefore, in many clinical situations, the use of GBCAs in children is considered off-label but is well-supported as a standard-of-care in clinical practice.

GBCAs have been used safely in the pediatric population since their introduction into clinical practice in the late $1980 \mathrm{~s}^{2-7} \mathrm{In}-$ deed, pediatric GBCA administration has been associated with lower incidences of acute adverse reactions than GBCA administration in adults, with a $0.04 \%$ frequency in children compared with $0.07 \%$ in adults. ${ }^{8}$ Furthermore, there are only a few case reports of children developing nephrogenic systemic fibrosis, a rare complication of GBCA administration associated with acute or severe chronic renal disease resulting in a scleroderma-like reaction of the skin along with systemic involvement of the internal organs. ${ }^{9}$ To our knowledge, there are no reported cases of nephrogenic systemic fibrosis in patients younger than 2 years of age despite the clinical use of GBCAs in this age group and the known renal immaturity of neonates. In these very young patients, the estimated glomerular filtration rate (milliliters/minute $/ 1.73 \mathrm{~m}^{2}$ ) values do not typically reach $60 \mathrm{~mL} / \mathrm{min} / 1.73 \mathrm{~m}^{2}$ until the patient is older than 3 months of age or later in preterm infants. ${ }^{10}$

Recently, however, there have been reports of gadolinium deposition in the brain and other tissues of adult patients with normal renal function. This was first recognized by Kanda et $\mathrm{al}^{11}$ as a progressive increase in intrinsic T1 signal in the dentate nucleus and globus pallidus on unenhanced T1-weighted imaging that correlated with the number of previously administered doses of GBCA. This initial report by Kanda et al was quickly followed by other collaborating studies in adult patients ${ }^{12-16}$ and by pathologic confirmation that the increasing T1-weighted signal within the dentate nucleus corresponded to areas of gadolinium deposition. ${ }^{17,18}$ It is unknown, however, whether the pediatric brain is also susceptible to gadolinium deposition, given differences in physiology and the smaller GBCA doses pediatric patients are typically administered based on weighted-adjusted dosing. Despite case reports of 2 pediatric patients, ${ }^{19,20}$ retrospective series have not been described in children. Here, we present a series of pediatric patients exposed to multiple doses of GBCAs who developed T1-weighted hyperintensity in the dentate nucleus, which correlated with the number of administered GBCA doses. We also introduce an automated method for ROI delineation to eliminate reader bias.

\section{MATERIALS AND METHODS}

The study was approved by the Medical University of South Carolina institutional review board and was compliant with the Health Insurance Portability and Accountability Act. Informed consent was waived due to the retrospective nature of the study.

\section{Patients}

To determine our total institutional pediatric GBCA exposure, we included all pediatric patients who had been administered any GBCA at our institution during an approximately 20-year period (January 1, 1995, to June 30, 2014). All patients who received at least 1 dose of a GBCA at our institution and who were 18 years of age or younger at the time of administration were included. Many of these patients had also received additional doses of GBCAs at outside institutions, which could not be verified; therefore, these gadolinium doses were not included in the calculated total number of documented GBCA administrations.

During the period investigated, the most widely used MR imaging contrast agent for pediatric MR imaging at our institution was gadopentetate dimeglumine (Magnevist; Bayer HealthCare Pharmaceuticals, Wayne, New Jersey), though gadodiamide (Omniscan; GE Healthcare, Piscataway, New Jersey), gadoteridol (ProHance; Bracco Diagnostics, Princeton, New Jersey), and gadobenate dimeglumine (MultiHance; Bracco Diagnostics) were also available at various times during the 20 -year period. To limit assessment to 1 agent, we included those patients who had received at least 5 consecutive doses of only Magnevist, as recorded by the MR imaging technologist at the time of the scan. We cannot, however, completely exclude the possibility that the patients received other agents such as an unknown GBCA at an outside institution.

Of the total pediatric population who had contrast-enhanced MR imaging examinations at our institution from January 1, 1995 , to June 30, 2014, we identified 58 pediatric patients who had undergone at least 5 consecutive MR imaging scans following a documented intravenous injection of gadopentetate dimeglumine. Thirty-six patients were excluded from analysis with any form of posterior fossa pathology involving the cerebellum, including cerebellar tumors, infection, or congenital malformations. Finally, 6 additional patients who had undergone posterior fossa radiation therapy were excluded. Radiation therapy was defined as whole-brain radiation or any radiation treatment that included the posterior fossa in the radiation field. Therefore, 16 patients meeting the inclusion criteria were analyzed. We screened these patients for any degree of renal failure as defined by an estimated glomerular filtration rate value of $<60 \mathrm{~mL} / \mathrm{min} / 1.73$ $\mathrm{m}^{2}$, liver failure, parenteral nutrition, or the presence of additional underlying disorders such as congenital metabolic diseases. Clinical information, imaging data, and the administered GBCA were obtained from the electronic medical records of our hospital, PACS, and the radiology information management system. See Table 1 for patient clinical information.

\section{MR Imaging Protocol}

All patients underwent MR imaging on either a 1.5T or 3T system. Various MR imaging systems were available for patient imaging during the 20-year period, including both Philips Healthcare (Best, the Netherlands) and Siemens (Erlangen, Germany). In all patients, the MR imaging protocol included a sagittal, unenhanced, T1-weighted spin-echo sequence (section thickness, 3-5 mm; gap, 1-2.5 mm; TR, 300-691 ms; TE, 8-20 ms). The inplane resolution ranged from 0.69 to $1.1 \mathrm{~mm}$, with most being $0.86 \mathrm{~mm}$ (matrix, $256 \times 256$ ). Most commonly, the sagittal T1weighted sequence was of the whole brain; however, occasionally, patients underwent imaging per a dedicated protocol (pituitary, face, or neck) that did not include whole-brain coverage. These scans were also evaluated if the reviewer deemed the sequence adequate for visualization of the dentate nucleus and cerebellar white matter. Gadopentetate dimeglumine was administered as an intravenous bolus by using body weighted-adjusted dosing at $0.1 \mathrm{mmol} / \mathrm{kg}$ per departmental protocol.

AJNR Am J Neuroradiol 37:2340-47 Dec 2016 www.ajnr.org 
Table 1: Clinical description and number of doses of GBCA for patients who underwent ROI analysis ${ }^{\mathrm{a}}$

\begin{tabular}{|c|c|c|c|c|c|}
\hline $\begin{array}{c}\text { Patient } \\
\text { No. }\end{array}$ & Diagnosis & Chemotherapy & $\begin{array}{c}\text { Total } \\
\text { Parenteral } \\
\text { Nutrition }\end{array}$ & $\begin{array}{l}\text { No. of Gabopentetate } \\
\text { Dimeglumine Doses } \\
\text { Prior to MRI Study } \\
\text { Evaluated by } \\
\text { Blinded Reader }\end{array}$ & $\begin{array}{l}\text { No. of MRI Studies } \\
\text { with GBCA } \\
\text { Administration } \\
\text { Evaluated by } \\
\text { Automated Analysis }\end{array}$ \\
\hline 1 & Temporal astrocytoma/glioblastoma & Yes & No & 12 & 14 \\
\hline 2 & Optic glioma/neurofibromatosis type 1 & No & No & 7 & NA \\
\hline 3 & Temporal desmoplastic infantile ganglioma & No & No & 7 & 6 \\
\hline 4 & Temporoparietal pleomorphic xanthoastrocytoma & Yes & No & 7 & 10 \\
\hline 5 & Craniopharyngioma & No & No & 7 & 4 \\
\hline 6 & Parieto-occipital desmoplastic infantile ganglioma & No & No & 4 & 11 \\
\hline 7 & Marfan syndrome with CSF leaks & No & No & 7 & 8 \\
\hline 8 & Parotid hemangiomas & No & No & 4 & 4 \\
\hline 9 & Multiple sclerosis & No & No & 5 & 5 \\
\hline 10 & Thalamic astrocytoma & No & No & 12 & 14 \\
\hline 11 & Parapharyngeal rhabdomyosarcoma & Yes & No & 7 & NA \\
\hline 12 & Maxillary sinus vascular malformation & No & No & 16 & 15 \\
\hline 13 & Brain stem encephalitis & No & No & 6 & 6 \\
\hline 14 & Thoracic spinal cord pilocystic astrocytoma & Yes & Yes & 7 & NA \\
\hline 15 & Parietal low-grade astrocytoma & Yes & No & 6 & 27 \\
\hline 16 & Frontal pleomorphic xanthoastrocytoma & Yes & No & 6 & 5 \\
\hline
\end{tabular}

Note:-NA indicates not applicable.

a None of the patients had documented renal failure. A few patients occasionally had mildly elevated liver function test levels, but none were diagnosed with liver failure.

\section{Image Analysis: User-Defined ROIs}

We used methods similar to those reported in the literature. ${ }^{11,21}$ For each patient, the last contrasted MR imaging scan with documented gadopentetate dimeglumine administration was evaluated. By visual inspection, a board-certified neuroradiologist, blinded to the patient's clinical history and the number of prior contrast administrations, placed ROIs within the dentate nucleus and the cerebellar white matter on unenhanced sagittal T1weighted images. ROIs were placed in the right dentate nucleus and in the adjacent cerebellar white matter on the same sagittal section. If the right dentate nucleus or cerebellar white matter was obscured by overlying artifacts, then ROIs were placed on the corresponding structures on the left. The mean signal intensity of each ROI was obtained and used to calculate dentate-to-cerebellar white matter (DN/C) ratios for each subject.

\section{Automated Image Analysis}

To eliminate reader bias, we also performed automated ROI placement. Of the 16 patients evaluated by the blinded reader, 3 patients had not undergone whole-brain imaging to fully cover the entire posterior fossa (MRIs performed per pituitary or neck protocols) and, therefore, were not included in automated analysis because complete coverage of the posterior fossa was required to perform image registration. Therefore, the sagittal, unenhanced T1-weighted sequences from 13 patients were subjected to automated analysis. Unlike the analysis by the blinded reader, all scans for each patient performed at our institution, available on the PACS and adequate for computer analysis, were included. In other words, the automated analysis was not limited to documented serial administrations of gadopentetate dimeglumine in order to increase our number of data points but was restricted to only those scans with whole-brain coverage. Therefore, the number of GBCA doses before the last MR imaging study examined by the blinded reader and the number of contrasted MR studies evaluated by automated analysis do not correspond (Table 1).

Image processing was performed by using the Spatially Un- biased Atlas Template of the Cerebellum and Brain Stem (SUIT; http://www.diedrichsenlab.org/imaging/suit_download. htm) toolbox, ${ }^{22,23}$ implemented in the statistical parametric mapping software package (SPM8; http://www.fil.ion.ucl.ac.uk/ $\mathrm{spm} /$ ). This toolbox uses a probabilistic atlas of the human cerebellum defined by the Montreal Neurological Institute (MNI) 152 template (http://neuro.debian.net/pkgs/fsl-mni152-templates. html) optimized for delineation of the deep cerebellar nuclei. ${ }^{23}$ Our procedure involved the following steps: 1) cropping and isolating the cerebellum and brain stem from the T1-weighted anatomic images, 2) normalizing each cropped image into SUIT space, 3) placing ROIs based on MNI 152 template coordinates, 4) reflecting the ROIs into individual subject space by using the deformation parameters from the normalization, 5) manually inspecting the results, and 6) calculating the median intensity value in each ROI.

The atlas provided by the SUIT toolbox is a probability atlas, so the dentate nucleus ROI was defined as voxels with $>75 \%$ probability of being within the dentate nucleus. Using this threshold, we found the mean volume of the dentate nucleus ROI to be $0.66 \mathrm{~mL}$, similar to the volume of the dentate nucleus as reported for high-resolution $3 \mathrm{~T}$ images. ${ }^{24}$

ROIs placed in the pons, cerebellar white matter, CSF, and cerebellar gray matter were investigated for potential use as normalization factors (Fig 1). Cerebellar gray matter (MNI coordinates: $\pm 20,-68,-50)$, cerebellar white matter of the middle cerebellar peduncles (MNI coordinates: $\pm 18,-40,-36$ ), CSF within the fourth ventricle (MNI coordinates: $0,-44,-30$ ), and the central pons (MNI coordinates: $0,-24,-32$ ) ROIs were defined in SUIT space as spheres of $7-\mathrm{mm}$ radii. The radius of the CSF ROI was $4 \mathrm{~mm}$ due to the size limitation of the fourth ventricle.

For each ROI, the median signal intensity was obtained. Scatterplots were constructed showing all dentate ROIs across all subjects and all scans versus those of the pons, cerebellar white mat- 


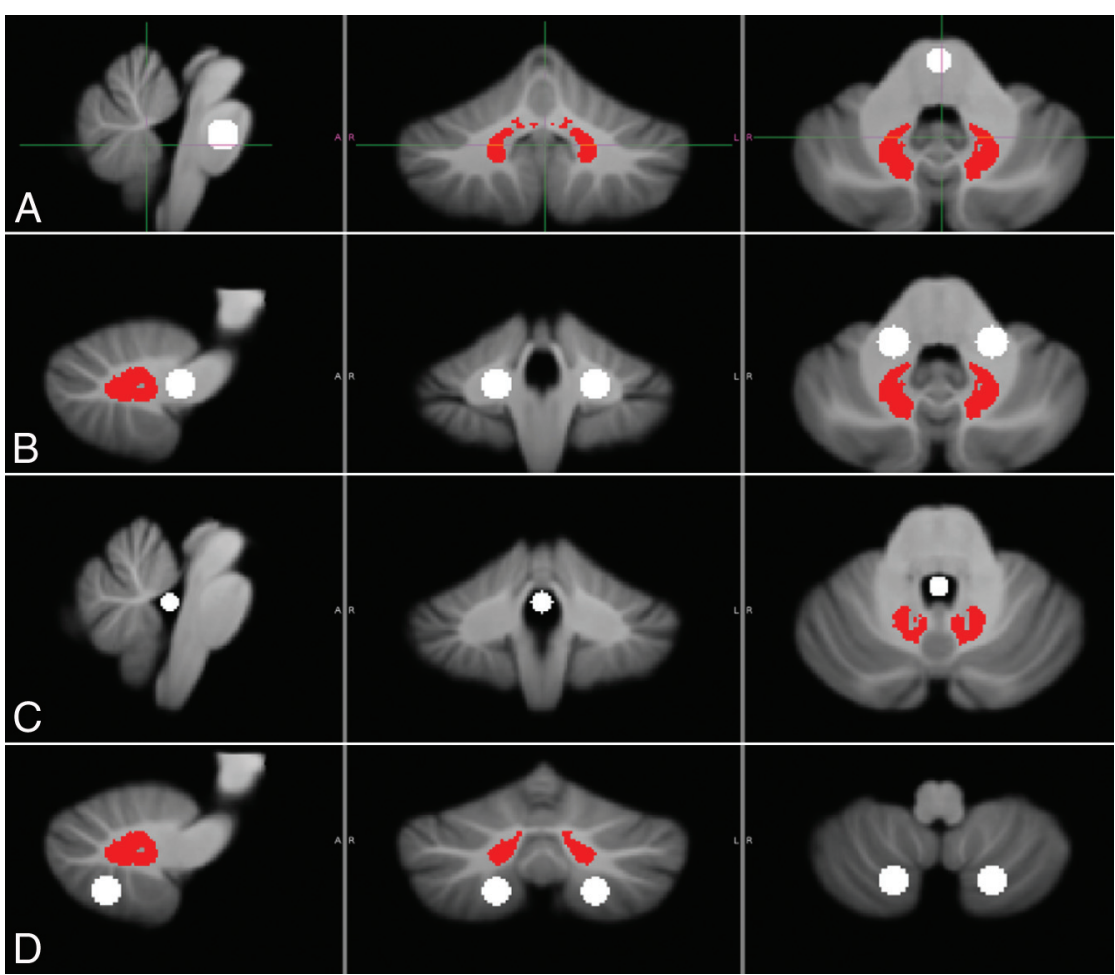

FIG 1. ROIs were defined by the SUIT template within the dentate nucleus (in red) and within the pons $(A)$, cerebellar white matter $(B), \operatorname{CSF}(C)$, and cerebellar gray matter $(D)$ (ROls in white) to serve as potential references for normalization.

Table 2: Total number of pediatric patients who underwent at least 1 contrast-enhanced MRI at our institution from January 1 , 1995, to June 30, 2014

\begin{tabular}{lc}
\hline No. of Doses GBCAs Administered & No. of Pediatric Patients \\
\hline 1 & 9306 \\
2 & 1488 \\
3 & 452 \\
4 & 183 \\
5 & 101 \\
6 & 48 \\
7 & 40 \\
8 & 19 \\
9 & 20 \\
10 & 10 \\
11 & 13 \\
12 & 8 \\
13 & 3 \\
14 & 2 \\
15 & 3 \\
16 & 5 \\
17 & 3 \\
18 & 1 \\
19 & 1 \\
20 & 1 \\
21 & 1 \\
38 & 1 \\
Total No. of pediatric patients & 1109 \\
\hline
\end{tabular}

ter, CSF within the fourth ventricle, and cerebellar gray matter. CSF was found to be the worst reference point for normalizing the dentate signal intensities because it was more susceptible to noise due to the low intensity of CSF on T1-weighted images. Cerebellar white matter and the pons appeared to be the best choices for a ratio-based reference because they exhibited the least noise in the dataset. The dentate-to-pons (DN/P) ratios and $\mathrm{DN} / \mathrm{C}$ ratios were then calculated for each subject for each MR imaging scan with an adequate sagittal T1weighted sequence available on our institutional PACS system.

\section{Statistical Analysis}

For the blinded reader's scores, the Spearman rank order correlation was used to identify associations among non-normally distributed variables, and 2-tailed $P$ values are reported. Sixteen subjects were included in the analyses, and the correlations between the number of doses and the DN/C ratios at the time of the last MR imaging scan were examined.

For statistical analysis of the automated scores, general linear mixed models were used. ${ }^{25}$ For each dependent variable (DN/P and DN/C ratios), a general linear mixed model was constructed by using the number of prior doses as the primary independent variable. To account for repeat measurements being obtained on the study subjects, we created several general linear mixed models for each dependent variable; the models involved different types of random effects (intercepts, slopes) and different types of residual correlation structures (eg, unstructured, compound symmetry, spatial power). A final model was selected for each dependent variable by comparing the Akaike information criteria and overall model parsimony. Analysis was performed by using SAS, Version 9.4 (SAS Institute, Cary, North Carolina).

\section{RESULTS}

Between January 1995 and June 2014 at our institution, 11,709 pediatric patients received at least 1 dose of a GBCA (Table 2). The maximum number of doses was 38 in 1 patient, though 16 patients received at least 15 doses and 280 patients received at least 5 doses of contrast. These data do not include additional GBCA doses received by some patients at outside institutions.

Sixteen patients met the inclusion/exclusion criteria for analysis. The patient ages ranged from 2 months to 14 years at the time of the first contrast dose (mean age, $7.4 \pm 4.8$ years). None of the patients had documented renal failure or abnormal renal function, with all measured estimated glomerular filtration rate values $>60 \mathrm{~mL} / \mathrm{min} / 1.73 \mathrm{~m}^{2}$. A few patients occasionally had mildly elevated liver function test results, but none were diagnosed with liver failure. One patient had undergone total parenteral nutrition for approximately 5 weeks during the imaging period. For 2 patients, unenhanced sagittal T1-weighted images were unavailable on our institutional PACS following the fifth dose of gadopentetate dimeglumine. For these 2 cases, we used ROI values obtained on the preceding MR imaging (following only 4 doses of gadopentetate dimeglumine). Therefore, the number of contrast 

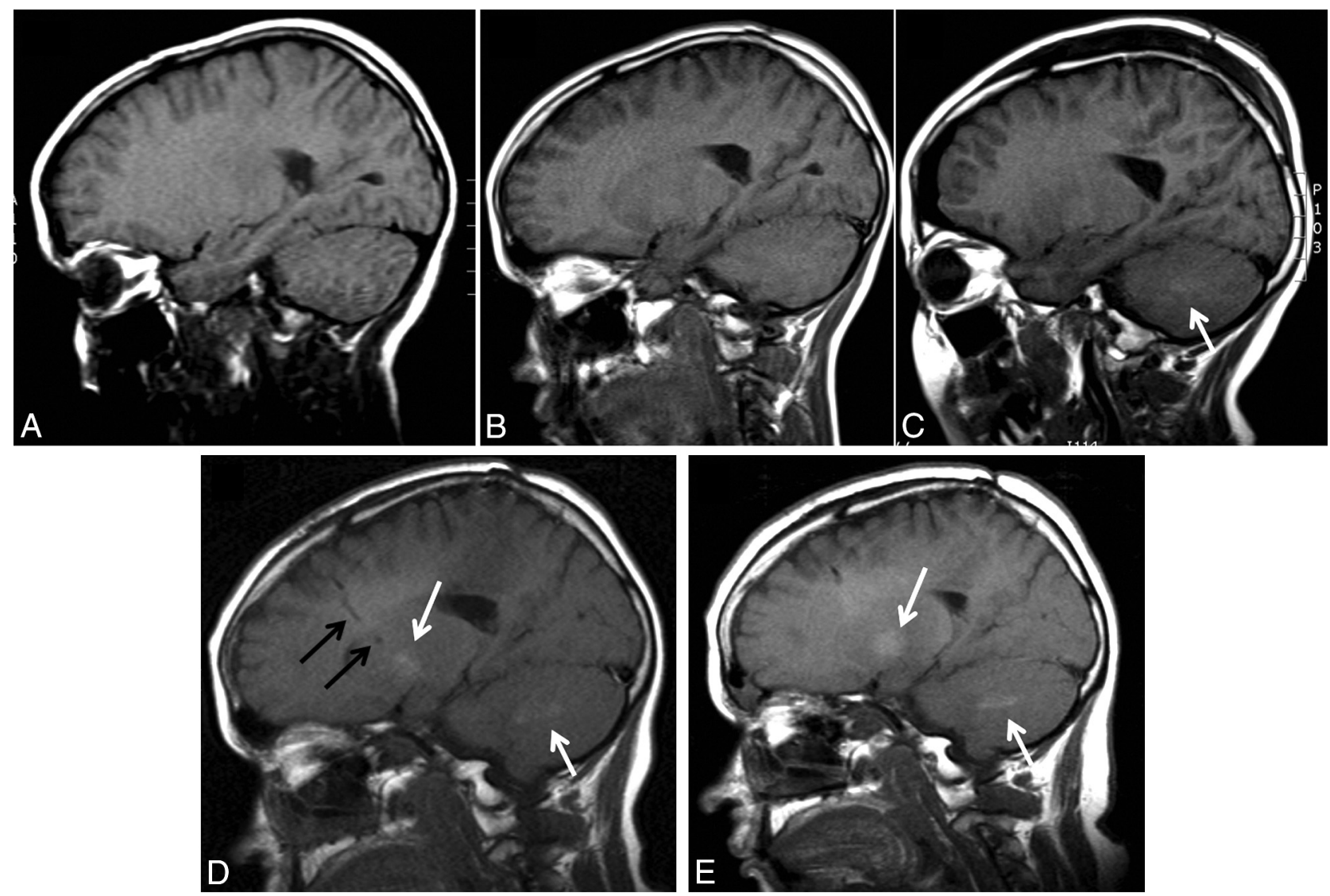

FIG 2. A, Sagittal unenhanced T1-weighted image from the initial MR imaging of a patient who presented with a parietal lobe low-grade astrocytoma at 10 years of age. B, Sagittal unenhanced T1-weighted image from an MR imaging at 13 years of age after 6 doses of GBCAs. C, Sagittal unenhanced T1-weighted image from an MR imaging following lesion biopsy at 13 years of age after 12 doses of GBCAs. There is faint hyperintensity within the dentate nucleus (white arrow). D. Sagittal unenhanced T1-weighted image from an MR imaging at 15 years of age after 17 doses of GBCAs. Black arrows indicate the tract of a ventricular catheter. There is distinct hyperintensity within the dentate nucleus and the globus pallidus (white arrows). E, Sagittal unenhanced T1-weighted image from an MR imaging scan at 17 years of age after 27 doses of GBCAs. Hyperintensity within the dentate nucleus and the globus pallidus is again seen (white arrows).

doses before the last brain MR imaging with gadopentetate dimeglumine examined by the blinded reader ranged from 4 to 16 .

Hyperintensity was visible within the dentate nucleus on unenhanced images in the patients in our series who had received at least 7 prior doses of GBCA (Fig 2). For blinded reader analysis, the prior number of doses of GBCA before the last scan was significantly associated with the $\mathrm{DN} / \mathrm{C}$ ratio $\left(r_{\mathrm{s}}=0.77, P=.001\right)$. As the number of doses increased, so did the DN/C ratio.

All computer-placed ROIs were confirmed by manual inspection (Fig 3). Figure 4 shows all DN/P and DN/C ratios plotted against the number of prior contrast doses for all time points for all 13 subjects who underwent automated analysis. Analysis of the computer-placed ROIs by using general linear mixed modeling demonstrated a significant correlation between the number of prior doses and both the DN/P ratio $(t=4.98, P<$ $.0001)$ and the DN/C ratio $(t=2.73, P<.02)$. Figure 5 shows the predicted mean values along with their $95 \%$ confidence bands. Note that only 16 prior contrast doses were plotted because only 1 subject contributed to measurements for $>16$ doses. Overall, as the number of doses increased with each scan, so did both the DN/P and DN/C ratios as detected by automated ROI analysis.

\section{DISCUSSION}

Several reports have now described the deposition of gadolinium in the brain of adult patients with normal renal function. ${ }^{11-18}$ Here we present a pediatric case series demonstrating a significant correlation between the $\mathrm{DN} / \mathrm{C}$ and the $\mathrm{DN} / \mathrm{P}$ ratios and the prior number of doses of GBCA administered. These data strongly support an association in children between increasing cumulative GBCA dose and hyperintensity within the dentate nucleus on unenhanced T1-weighted imaging, a finding shown by pathologic confirmation in adults to correspond to areas of gadolinium deposition. ${ }^{17,18}$

Improved imaging, along with advances in neurosurgery, radiation oncology, and chemotherapy, has contributed to improvement in the cure rates of childhood cancers during the past 2 decades, and ongoing advances in genomics technologies are expected to dramatically transform pediatric neuro-oncology. ${ }^{26}$ The improved cure rates of childhood cancers and longer term survival have led to increasing numbers of children exposed to greater cumulative doses of GBCAs. In our study, the maximum number of doses was 38 in 1 patient, though 280 pediatric patients received at least 5 doses of contrast.

Pathologic evaluation of the brain in patients with normal 

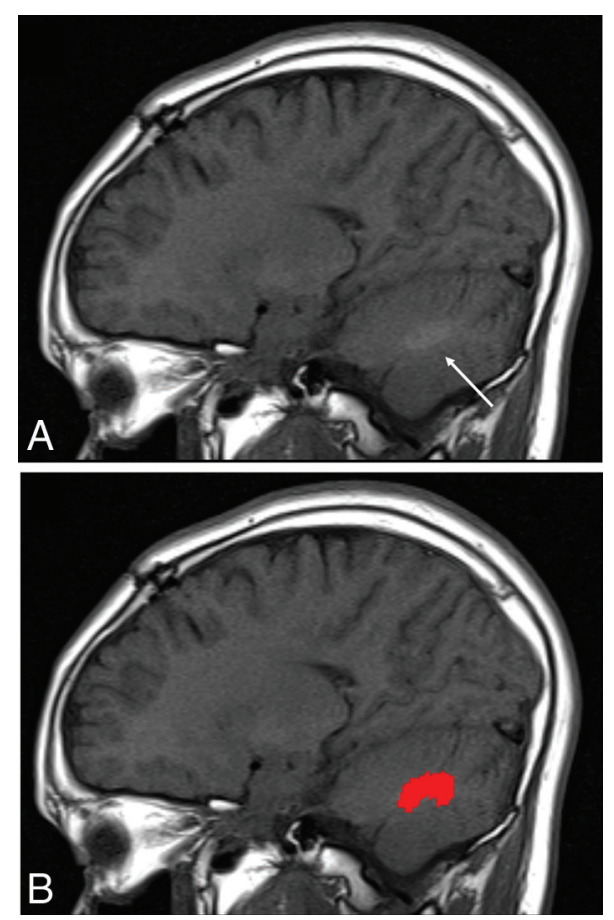

FIG 3. A, Sagittal unenhanced Tl-weighted image from an MR imaging of a patient at 13 years of age after the 12th dose of GBCA. An arrow marks the location of the dentate nucleus. $B, A$ computer-assigned dentate $\mathrm{ROI}$ reflected back into the individual patient's space.
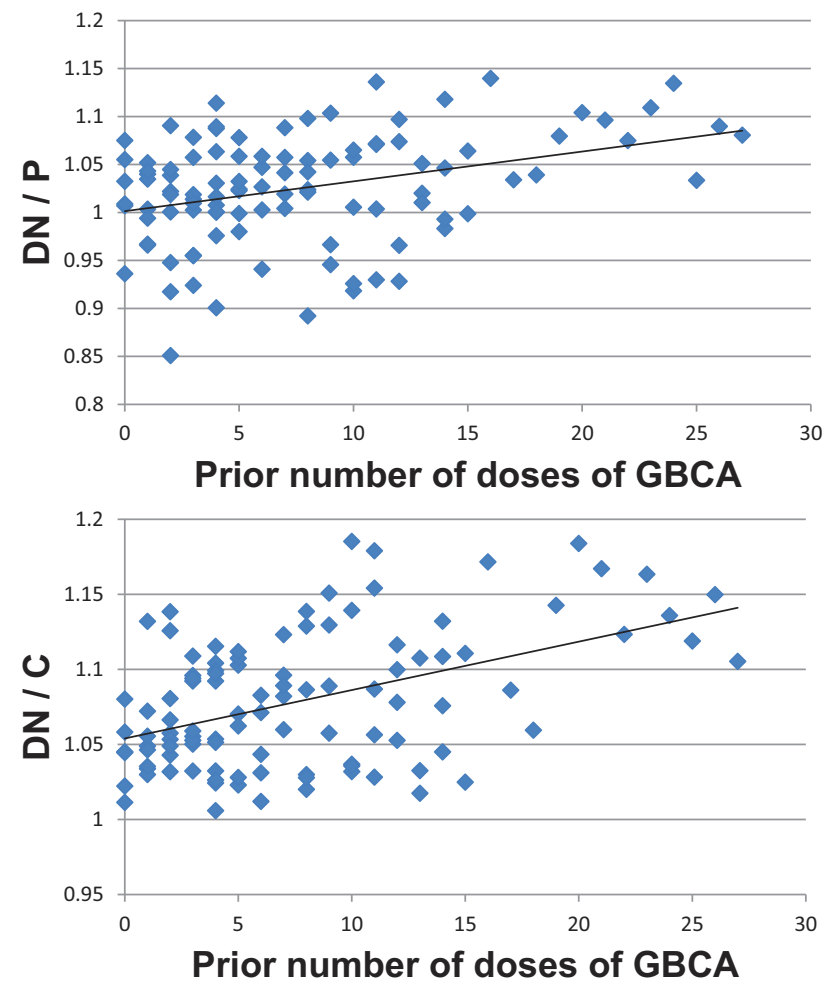

FIG 4. Computer-selected $D N / P$ and $D N / C$ ratios plotted against the number of prior contrast doses for all time points for all 13 subjects who underwent automated analysis.

renal function who were administered GBCAs has shown that gadolinium is deposited not only in the dentate nucleus but throughout the brain, including the frontal lobe white matter and frontal cortex. ${ }^{17,18,27}$ While the clinical significance of the longterm retention of gadolinium in the brain is unknown, it is particularly concerning for pediatric patients, who are undergoing neurodevelopment. Brain development begins during fetal life and continues throughout adolescence with prefrontal, posterior parietal, and other high-order association areas being the last areas to undergo myelination. ${ }^{28}$ There is a dramatic proliferation of synapses in the prefrontal cortex during early and mid-childhood, reaching numbers that exceed adult levels by 2 - or 3-fold during puberty, followed by a plateau phase during adolescence with subsequent elimination and reorganization of prefrontal synaptic connections. ${ }^{28}$ During this critical period of development, the brain is particularly vulnerable to toxin exposure, ${ }^{29}$ and it is unknown whether the processes of active myelination, growth, and neuronal pruning could be affected by exposure to gadolinium deposition. Further research is needed to address the clinical significance, if any, of gadolinium deposition in the body of pediatric patients.

In a study measuring total gadolinium deposition by using inductive coupled plasma mass spectroscopy, Murata et $\mathrm{al}^{27} \mathrm{dem}-$ onstrated the presence of gadolinium within the brain, skin, and bones of adult patients following the administration of 1-11 doses of GBCA. In their study, the gadolinium levels within the bones were 23 times higher than those in the brain, ${ }^{27}$ suggesting that gadolinium deposition in the brain may represent a marker for even higher levels of gadolinium stored in the bones. This finding is particularly concerning for pediatric patients exposed to high cumulative doses of GBCAs during skeletal ossification and periods of rapid bone growth. It has been hypothesized that high levels of gadolinium storage in the bones could represent an internal source of gadolinium exposure throughout a patient's lifetime, with the mobilization of gadolinium later in life during conditions favoring calcium mobilization from bones (renal failure, osteoporosis, long-term bed rest, hyperparathyroidism, and pregnancy). ${ }^{30}$

Currently, there are 9 GBCAs approved for use in the United States. Each commercially available GBCA is composed of gadolinium along with a ligand molecule whose structure varies among the agents. There are 2 distinct categories of GBCAs based on the structure of the ligand: the "macrocyclic" molecules, in which the gadolinium ion is "caged" into a preorganized cavity of the ligand, and the "open-chain linear" chelates, in which the metal ion is attached to the end of the ligand molecule. Gadolinium chelates can also be either nonionic or ionic. These structural differences result in differing thermodynamic and kinetic stabilities, with the highest thermodynamic and kinetic stability achieved by an ionic, macrocyclic structure. ${ }^{31}$ Recent reports suggest that the stability of the GBCA may play a role in the deposition of gadolinium in the brain because administration of linear agents is associated with hyperintensity in the dentate nucleus, while administration of macrocyclic agents has not shown this association. ${ }^{14,21}$ Higher stability, macrocyclic agents have been shown to be associated with lower levels of gadolinium deposition in the body in both human and animal studies. ${ }^{32,33}$ Given the current uncertainties concerning the clinical significance of gadolinium deposition within the body, in pediatric imaging, we would recommend taking a cautious stance and considering the

AJNR Am J Neuroradiol 37:2340-47 Dec 2016 www.ajnr.org

2345 

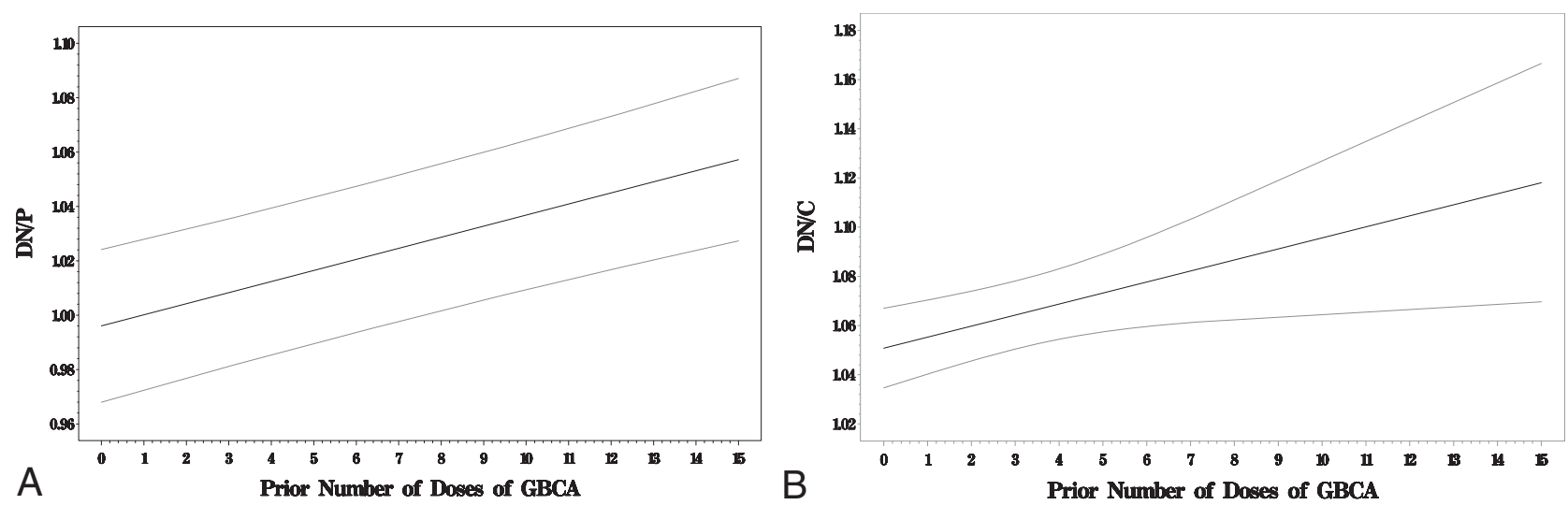

FIG 5. A, Association between DN/P ratio and the number of prior doses of GBCA. The dark solid line reflects the predicted average response among subjects' median DN/P ratio values at the given number of prior doses of GBCA, and the light solid lines represent the associated $95 \%$ confidence band. B, Association between the DN/C ratio and the number of prior doses of GBCA. The dark solid line reflects the predicted average response among subjects' median DN/C ratio values at the given number of prior doses of GBCA, and the light solid lines represent the associated $95 \%$ confidence band.

use of only higher stability, macrocyclic agents in pediatric patients, given their unique vulnerability, while awaiting the results of long-term research studies to address any potential clinical significance of gadolinium deposition in the body.

Our study had several potential sources of error. The MR imaging scans were acquired using various $\mathrm{MR}$ imaging systems and scanning parameters. A single GBCA was not used consistently for all patients, and outside GBCA doses could not be verified. Agematched controls were not included. For the subjectively placed ROIs, sources of error include image noise resulting in large variations in ROI values and reader bias. Despite our reader being blinded, the clearly visible hyperintense appearance of the dentate nucleus in patients with larger cumulative GBCA doses could have influenced ROI placement. Sources of error in the automated ROI analysis include areas of artifacts overlying the dentate nucleus, cerebellar white matter, or pons, such as flow artifacts from the transverse sinuses and metallic artifacts from implanted hardware. Our reader excluded these areas visually during ROI placement; however, these areas marred by artifacts could not be omitted by the image-processing software. Errors in the registration process cannot be excluded though all computer-placed ROIs were confirmed by manual inspection. Differences in white matter myelination could be a confounding variable in the automated ROI analysis in the youngest age groups; however, only 2 of the 16 patients were younger than 1 year of age at the time of the first scan and both were older than 2 years of age at the time of their last scan. Despite the limitations of using an automated method for delineation of the dentate nucleus, pons, and cerebellar white matter, we believe the advantage of eliminating reader bias is important due to the large variation in ROI values obtained with only slight adjustment of ROI placement.

\section{CONCLUSIONS}

As in the adult population, pediatric patients also demonstrate progressive $\mathrm{T} 1$-weighted hyperintensity in the dentate nucleus correlating with the number of administered doses of GBCA, suggesting a causal relationship between gadolinium administration and the signal changes seen on MR imaging. Confirmation of gadolinium deposition within the pediatric brain requires biopsy confirmation as was demonstrated in the adult population. The clinical significance of long-term retention of gadolinium in the developing brain is currently unknown. Therefore, pediatric imagers should continue to use prudence in selecting patients to undergo contrasted MR imaging and in selecting the appropriate contrast agents to use in this population, particularly in patients requiring multiple contrast exposures. A risk-benefits analysis, incorporating the potential for long-term gadolinium deposition in brain tissue should be considered in the decision-making process for all pediatric patients. When a decision is made to proceed with contrast administration in a pediatric patient, the specific agent administered, the dose, and the cumulative dose should always be recorded for each examination. Most important, a patient should not be deprived of a well-indicated contrasted MR examination.

\section{ACKNOWLEDGMENTS}

The authors acknowledge the contributions of Nancy J. Monogan, Alden Finlayson, R.T. (R) (MR), and Sarah L. Brewer in preparing the data for this article.

Disclosures: Donna R. Roberts—RELATED: Grant: National Institutes of Health*; UNRELATED: Board Membership: Guerbet, Comments: I serve as an advisory board member and consultant for Guerbet; Consultancy: Guerbet, Comments: I serve as an advisory board member and consultant for Guerbet; Other: Guerbet, ${ }^{*}$ Comments: I am a site principal investigator for a current clinical trial sponsored by Guerbet and have been a site principal investigator for previous Guerbet-sponsored clinical trials; OTHER: I am a member of the FDA Medical Imaging Drugs Advisory Committee and serve on the FDA advisory panel recommending approval for the gadobutrol contrast agent. Genevieve Bolles-RELATED: Grant: National Institutes of Health.* Paul J. Nietert-RELATED: Grant: National Institutes of Health (grant ULITR001450).* *Money paid to the institution.

\section{REFERENCES}

1. American College of Radiology. ACR Appropriateness Criteria. http://www.acr.org/Quality-Safety/Appropriateness-Criteria. Accessed March 14, 2016

2. Balassy C, Roberts D, Miller SF. Safety and efficacy of gadoteric acid in pediatric magnetic resonance imaging: overview of clinical trials and post-marketing studies. Pediatr Radiol 2015;45:1831-41 CrossRef Medline

3. Ball WS Jr, Nadel SN, Zimmerman RA, et al. Phase III multicenter 
clinical investigation to determine the safety and efficacy of gadoteridol in children suspected of having neurologic disease. Radiology 1993;186:769-74 CrossRef Medline

4. Elster AD. Cranial MR imaging with Gd-DTPA in neonates and young infants: preliminary experience. Radiology 1990;176:225-30 CrossRef Medline

5. Hahn G, Sorge I, Gruhn B, et al. Pharmacokinetics and safety of gadobutrol-enhanced magnetic resonance imaging in pediatric patients. Invest Radiol 2009;44:776-83 CrossRef Medline

6. Lundby B, Gordon P, Hugo F. MRI in children given gadodiamide injection: safety and efficacy in CNS and body indications. Eur J Radiol 1996;23:190-96 CrossRef Medline

7. Schneider G, Schürholz H, Kirchin MA, et al. Safety and adverse effects during 24 hours after contrast-enhanced MRI with gadobenate dimeglumine (MultiHance) in children. Pediatr Radiol 2013; 43:202-11 CrossRef Medline

8. Dillman JR, Ellis JH, Cohan RH, et al. Frequency and severity of acute allergic-like reactions to gadolinium-containing i.v. contrast media in children and adults. AJR Am J Roentgenol 2007;189: 1533-38 CrossRef Medline

9. Girardi M, Kay J, Elston DM, et al. Nephrogenic systemic fibrosis: clinicopathological definition and workup recommendations. J Am Acad Dermatol 2011;65:1095-1106.e7 CrossRef Medline

10. Cooper S. Nephrology. In: Tschudy MM, Arcara KM, eds. The Harriet Land Handbook. 19th ed. Philadelphia: Elsevier Mosby; 2012:476-503

11. Kanda $\mathrm{T}$, Ishii $\mathrm{K}$, Kawaguchi $\mathrm{H}$, et al. High signal intensity in the dentate nucleus and globus pallidus on unenhanced T1-weighted MR images: relationship with increasing cumulative dose of a gadolinium-based contrast material. Radiology 2014;270:834-41 CrossRef Medline

12. Errante Y, Cirimele V, Mallio CA, et al. Progressive increase of T1 signal intensity of the dentate nucleus on unenhanced magnetic resonance images is associated with cumulative doses of intravenously administered gadodiamide in patients with normal renal function, suggesting dechelation. Invest Radiol 2014;49:685-90 CrossRef Medline

13. Quattrocchi CC, Mallio CA, Errante Y, et al. Gadodiamide and dentate nucleus $\mathrm{T} 1$ hyperintensity in patients with meningioma evaluated by multiple follow-up contrast-enhanced magnetic resonance examinations with no systemic interval therapy. Invest Radiol 2015; 50:470-72 CrossRef Medline

14. Radbruch A, Weberling LD, Kieslich PJ, et al. Gadolinium retention in the dentate nucleus and globus pallidus is dependent on the class of contrast agent. Radiology 2015;275:783-91 CrossRef Medline

15. Radbruch A, Weberling LD, Kieslich PJ, et al. High-signal intensity in the dentate nucleus and globus pallidus on unenhanced T1weighted images: evaluation of the macrocyclic gadolinium-based contrast agent gadobutrol. Invest Radiol 2015;50:805-10 CrossRef Medline

16. Weberling LD, Kieslich PJ, Kickingereder P, et al. Increased signal intensity in the dentate nucleus on unenhanced T1-weighted images after gadobenate dimeglumine administration. Invest Radiol 2015;50:743-48 CrossRef Medline

17. Kanda T, Fukusato T, Matsuda M, et al. Gadolinium-based contrast agent accumulates in the brain even in subjects without severe renal dysfunction: evaluation of autopsy brain specimens with induc- tively coupled plasma mass spectroscopy. Radiology 2015;276: 228-32 CrossRef Medline

18. McDonald RJ, McDonald JS, Kallmes DF, et al. Intracranial gadolinium deposition after contrast-enhanced MR imaging. Radiology 2015;275:772-82 CrossRef Medline

19. Roberts DR, Holden KR. Progressive increase of T1 signal intensity in the dentate nucleus and globus pallidus on unenhanced T1weighted MR images in the pediatric brain exposed to multiple doses of gadolinium contrast. Brain Dev 2016;38:331-36 CrossRef Medline

20. Miller JH, Hu HH, Pokorney A, et al. MRI brain signal intensity changes of a child during the course of 35 gadolinium contrast examinations. Pediatrics 2015;136:e1637-40 CrossRef Medline

21. Kanda $\mathrm{T}$, Osawa $\mathrm{M}$, Oba $\mathrm{H}$, et al. High signal intensity in dentate nucleus on unenhanced T1-weighted MR images: association with linear versus macrocyclic gadolinium chelate administration. $R a$ diology 2015;275:803-09 CrossRef Medline

22. Diedrichsen J, Balsters JH, Flavell J, et al. A probabilistic MR atlas of the human cerebellum. Neuroimage 2009;46:39-46 CrossRef Medline

23. Diedrichsen J, Maderwald S, Küper M, et al. Imaging the deep cerebellar nuclei: a probabilistic atlas and normalization procedure. Neuroimage 2011;54:1786-94 CrossRef Medline

24. Deoni SC, Catani M. Visualization of the deep cerebellar nuclei using quantitative $\mathrm{T} 1$ and rho magnetic resonance imaging at 3 Tesla. Neuroimage 2007;37:1260-66 CrossRef Medline

25. Fitzmaurice GM, Laird NM, Ware JH. Applied Longitudinal Analysis. Hoboken: Wiley-Interscience; 2004

26. Gajjar A, Bowers DC, Karajannis MA, et al. Pediatric brain tumors: innovative genomic information is transforming the diagnostic and clinical landscape. J Clin Oncol 2015;33:2986-98 CrossRef Medline

27. Murata N, Gonzalez-Cuyar LF, Murata K, et al. Macrocyclic and other non-group 1 gadolinium contrast agents deposit low levels of gadolinium in brain and bone tissue: preliminary results from 9 patients with normal renal function. Invest Radiol 2016;51:447-53 CrossRef Medline

28. Blakemore SJ. Imaging brain development: the adolescent brain. Neuroimage 2012;61:397-406 CrossRef Medline

29. Stein J, Schettler T, Wallinga D, et al. In harm's way: toxic threats to child development. J Dev Behav Pediatr 2002;23:S13-22 CrossRef Medline

30. Abraham JL, Thakral C. Tissue distribution and kinetics of gadolinium and nephrogenic systemic fibrosis. Eur J Radiol 2008;66: 200-07 CrossRef Medline

31. Port M, Idée JM, Medina C, et al. Efficiency, thermodynamic and kinetic stability of marketed gadolinium chelates and their possible clinical consequences: a critical review. Biometals 2008;21:469-90 CrossRef Medline

32. Robert $\mathrm{P}$, Violas $\mathrm{X}$, Grand $\mathrm{S}$, et al. Linear gadolinium-based contrast agents are associated with brain gadolinium retention in healthy rats. Invest Radiol 2016;51:73-82 CrossRef Medline

33. White GW, Gibby WA, Tweedle MF. Comparison of Gd(DTPABMA) (Omniscan) versus Gd(HP-DO3A) (ProHance) relative to gadolinium retention in human bone tissue by inductively coupled plasma mass spectroscopy. Invest Radiol 2006;41:272-78 CrossRef Medline 